\title{
Pharmacotherapy in patients with epilepsy and psychosis
}

1 Bertrand de Toffol, 2 Michael Trimble M, 3 Dale C Hesdorffer, 4,5 Lauren Taylor, 4,5 Perminder Sachdev, 6 Maurice Clancy, 7 Naoto Adachi, 8 José Augusto Bragatti, 9 Marco Mula, 10 Kousuke Kanemoto

1. Service de Neurologie \& Neurophysiologie Clinique, CHU Bretonneau, Tours, France, Service de Neurologie Hôpital de Cayenne, Guyane France et UMR 1253, iBrain, Université de Tours, Inserm

2. Institute of Neurology, Queen Square, London WC1N 3BG, United Kingdom

3. Gertrude H.K Sergievsky Center and Department of Epidemiology, Columbia University, USA

4. Centre for Healthy Brain Ageing, School of Psychiatry, University of New South Wales, Sydney, Australia.

5. Neuropsychiatric Institute, Prince of Wales Hospital, Randwick, NSW, Australia

6. Department of Liaison Psychiatry, University Hospital Waterford, Ireland

7. Adachi Clinic, Japan

8. Hospital de Clínicas de Porto Alegre, Division of Neurology, Porto Alegre, Brazil

9. Atkinson Morley Regional Neuroscience Centre, St George's University Hospitals NHS Foundation Trust and Institute of Medical and Biomedical Education, St George's University of London, United Kingdom

10. Aichi Medical University, Neuropsychiatric department, Nagakute, Japan 
Corresponding author: Bertrand de Toffol, Service de Neurologie et de Neurophysiologie Clinique, CHU Bretonneau, 2 bis Bvd Tonnellé 37044 Tours

Cedex France. Email : bertrand.detoffol@univ-tours.fr

Declarations of interest: none

This research did not receive any specific grant from funding agencies in the public, commercial, or not-for-profit sectors. 


\section{Abstract}

The recognition and treatment of psychosis in persons with epilepsy (PWE) is recommended with the apparent dilemma between treating psychosis and opening the possibility of exacerbating seizures. The pooled prevalence estimate of psychosis in PWE is 5.6\%. It has been proposed that a 'two hit' model, requiring both aberrant limbic activity and impaired frontal control, may account for the wide range of clinical phenotypes. The role of anticonvulsant drugs in psychosis in PWE remains unclear. Alternating psychosis, the clinical phenomenon of a reciprocal relationship between psychosis and seizures, is unlikely to be an exclusively anticonvulsant-specific phenomenon but rather, linked to the neurobiological mechanisms underlying seizure control. Re-evaluation of anticonvulsant treatment, including the agent/s being used and degree of epileptic seizure control is recommended. The authors found very few controlled studies to inform evidence-based treatment of psychosis in PWE. However, antipsychotics and benzodiazepines are recommended as the symptomatic clinical treatments of choice for postictal and brief interictal psychoses. The general principle of early symptomatic treatment of psychotic symptoms applies in epilepsy-related psychoses, as for primary psychotic disorders. In the authors' experience, low doses antipsychotic medications do not significantly increase clinical risk of seizures in PWE 
being concurrently treated with an efficacious anticonvulsant regimen.

\section{Key words}

Psychosis, epilepsy, seizures, antiepileptics, anticonvulsants, antipsychotics, postictal psychosis, interictal psychosis, alternating psychosis 


\section{Introduction}

Rational pharmacological treatment of psychoses in PWE should ideally be based upon the mechanisms linking the two disorders. However, the neurobiological understanding of these mechanisms remains incomplete, with absence of a satisfactory explanatory model to guide drug treatment. The empirical evidence is also insufficient. These guidelines for the treatment of Psychoses in PWE (Figure 1) are therefore based on the cumulative clinical experience of the authors and some systematic study. A two-step procedure, not necessarily consecutive, is recommended. The first step requires reevaluation of the antiepileptic treatment, an integral and under-recognized component of treatment in the authors' view. The second step requires initiation of pharmacotherapy including antipsychotic drugs (APD) and benzodiazepines. In this review, we discuss contextually-relevant translational neuroscience in Psychoses in PWE, recommended pharmacotherapy, and closely related topics such as alternating psychosis and the impact on seizure threshold of APD. Whilst the level of evidence remains low, partly due to challenges inherent in performing multisite randomized control trials, prompt empirically based treatment of patients with psychosis is strongly recommended. Untreated psychotic episodes in PWE can have devastating effects on the patients and those supporting them [1]

\section{Clinical classification of Psychoses in PWE}

Psychotic disorders are defined by the presence of delusions, hallucinations, disorganized thinking, grossly disorganized and/or abnormal motor behavior, and may include negative symptoms (DSM-5). Consciousness is typically preserved. Psychoses in PWE 
can be clinically characterized into two main categories depending upon the temporal relationship of symptom emergence to seizures: Interictal Psychosis (IIP) and Postictal Psychosis (PIP) [2]. IIP refers to psychosis that occurs in clear consciousness in PWE with temporal onset not during or immediately following a seizure. A personal or family history of psychosis, intellectual disability, and the sum of previous seizures are recognized vulnerability factors [3]. IIP can be further sub-classified according to duration (e.g., acute/brief/transient or chronic) and presumed etiology (e.g., drug-induced or forced normalization); however, these sub-classifications have not been validated. An individual with epilepsy may exhibit differing forms of IIP at different time points, e.g., drug-induced or non-drug induced, and acute or chronic [2]. Several epilepsy-related variables are considered pathogenically relevant in IIP including epilepsy type and seizure characteristics. Persons with focal epilepsy, particularly those who have temporal lobe seizures with impaired awareness, have a higher risk of IIP than those with generalized epilepsy [3]. Antiepileptic drugs (AEDs) and seizure frequency are also likely to have a significant association in the development of IIP. Altering the AED may consequently prevent or treat IIP in some cases.

Postictal psychosis (PIP) is a specific syndrome with clear temporal relationship between the acute onset of psychotic symptoms and the precipitating bout of focal 
impaired awareness seizures or generalized seizures. There is currently no diagnostic definition for PIP in DSM-5 and it would be coded as "Psychotic disorder due to another medical condition". The following specific diagnostic criteria have however been proposed: (1) Onset of psychosis within 1 week post seizure(s); (2) Psychosis duration exceeding 15 hours but less than 2 months; (3) Presence of one or more of the following: delusions, hallucinations, impaired consciousness, bizarre or disorganized behavior, formal thought disorder or affective changes; and (4) No evidence of AED toxicity, non-convulsive status epilepticus, recent head trauma, alcohol, or drug intoxication or withdrawal or chronic psychotic disorder that would better account for the current clinical presentation [4]. The presence of a lucid interval between the last seizure and the onset of psychotic symptoms rules out a simple confusional state. The clinical course of PIP is typically characterized by remission of the psychotic symptoms over several days (mean: 1 week) [5], with or without any treatment. Pre-psychotic EEG abnormalities persist during the psychosis. Risk factors for PIP include: long standing focal epilepsy, extratemporal onset, bilateral epileptiform activity, secondary generalization, slowing of the EEG background activity, and personal or family history of psychiatric disorders [6]. Brain MRI frequently shows structural abnormalities [6]. 
In order to understand the relationship between seizures and psychosis, PIP, IIP and alternating psychoses (in which an altered mental state arises with seizure frequency decrease or even control of the seizures) require a strong neurobiological model which is still lacking [1,7].

\section{Epidemiology of Psychoses in PWE}

In community-based studies of epilepsy that have examined the prevalence of psychosis, $3.1 \%$ to $9.2 \%[8,9,10,11]$ have been reported to have psychosis; $0.7 \%$ to $3.3 \%$ of all PWE have affective psychosis, $[10,12]$ and $0.7 \%$ to $1.2 \%$ have schizophrenia-like psychosis $[8,9,10,11]$. A systematic study demonstrated that psychotic symptoms (hallucinations and delusions in full consciousness which do not occur as seizure symptoms) are present in about $5.6 \%$ of patients with epilepsy (overall pooled prevalence) [13]. When PWE are compared to controls, psychotic disorders are 3-fold or more common in prevalent epilepsy $[10,11]$. Studies examining the temporal relationship between psychosis/schizophrenia and epilepsy have been conducted in large population-based registries because both disorders are relatively rare. The cumulative data suggest a bidirectional relationship between incident epilepsy and incident psychosis. Psychosis was associated with an increased risk for developing epilepsy in a Swedish study of hospitalized psychosis $(\mathrm{OR}=2.7 ; 95 \%$ CI 1.6-4.8) (14). In a UK general practice research database [15], psychosis was associated with a statistically significant increased risk for developing epilepsy in each of the three years before epilepsy onset (IRR=15.7, 8.5 and 7.7 in the $3^{\text {rd }}-1^{\text {st }}$ year before epilepsy onset).

In the same UK study [15], incident epilepsy was associated with a statistically significant increased risk of developing a first ever psychosis in each of the three years 
after epilepsy onset (IRR=10.9, 4.8 and 4.0 respectively). Similar results were seen when epilepsy of unknown etiology was examined. In two record linkage studies in Denmark, the incidence of non-organic non-affective psychoses was examined, excluding intellectual disability and/or substance misuse [16]. The standardized incidence ratio was significantly increased 1.48-fold for schizophrenia (95\% CI: 1.12-1.92), 2.04-fold for non-affective psychosis (95\% CI: 1.69-2.45) and 2.30-fold for non-organic non-affective psychoses (95\% CI: 2.02-2.59). There were no differences by gender or seizure type. A second Danish registry study compared epilepsy to controls [17]. Epilepsy was associated with a 2.18-fold increased risk for schizophrenia (95\% CI: 2.20-2.80) in the absence of a family history of psychosis, an important exclusion because family history is one of the established factors for an increased risk for schizophrenia in PWE [13]. The risk for schizophrenia-like psychosis was increased 2.93-fold (95\% CI: 2.69-3.20). There was no gender difference but increasing age was associated with a statistically significant increased risk for developing schizophrenia (Relative Risk: 2.03, 2.28, and 4.00 for people aged 15-24, 25-34 and $>35$ years), suggesting that the median age at onset of schizophrenia after epilepsy is greater than the onset of 22 years reported in the general population [18]. In families with a history of psychosis and a family history of epilepsy, there was an increased risk for schizophrenia and schizophrenia-like psychosis.

In addition, several population-based studies have suggested the existence of a bidirectional relationship between epilepsy and several psychiatric disorders including, ADHD, and primary mood, anxiety, and psychotic disorders. Not only are PWE at higher risk of developing one of these psychiatric disorders, but patients with one of these primary psychiatric disorders are at greater risk of developing epilepsy. This phenomenon is of great importance in the management of these psychiatric comorbidities, as the 
occurrence of seizures in a patient treated with psychotropic drug is not necessarily drug-related [19]. Although, in epidemiological terms, the relationship between epilepsy and psychosis might be considered to be bidirectional, this makes no assumption about the causation; in at least some cases it is likely that both conditions result from a common underlying cause, although whether that cause might be genetic, environmental or some combination of factors remains to be elucidated.

\section{Neurobiology of Psychoses in PWE}

Whilst explanatory models still remain speculative, there is some evidence that supports aberrant biochemical and neuroanatomic processes in the epileptic psychoses. However, it is uncertain whether such processes reflect pathophysiological cause, effect or a common aberrant substrate, given the cross-sectional nature of available data. A recent systematic review [20] found the following factors to be consistently associated with psychosis in temporal lobe epilepsy (TLE): early onset of epilepsy; history of status epilepticus, often nonconvulsive; unilateral or bilateral hippocampal sclerosis; cell loss in the CA1 hippocampal region; left hippocampal abnormalities. The left hippocampal abnormalities were specifically reduced $\mathrm{N}$-acetylaspartate (NAA, an intraneuronal amino acid utilised as a proxy measure of cerebral damage) and gray matter, (this association is also reported in non-epileptic psychotic disorders) and more impaired current cognitive performance. It is noteworthy that reports from intracranial depth electrode monitoring support the assertion that PIP occurs in a true postictal state, rather than reflecting subclinical persistent epileptogenic activity in deep brain structures [21].

Dysfunction in limbic networks has been widely hypothesized as the anatomical substrate for psychosis. However, more widespread cerebral changes are likely to be required for its development. Prefrontal dysfunction with inadequate inhibitory 
modulation of limbic activity in schizophrenic psychosis has been supported by animal models [22].

It has been speculated that frontal lobe structure, function and intact frontal-limbic networks might be of etiological importance in epileptic psychoses. Perfusion SPECT studies have demonstrated abnormalities in frontal and temporal regions in PIP suggesting that neuronal network dysfunction remote from the epileptogenic region is etiologically relevant [23,24]. A 'two hit' model of psychosis in epilepsy, requiring both aberrant limbic activity and impaired frontal control, may explain some of the wide range in clinical phenotypes of epileptic psychoses [25].

The repeated release of neurotransmitters, including acetylcholine, catecholamines, serotonin, opiates, adenosine and nitrous oxide during seizures is likely to pathogenically alter the function of postsynaptic receptors in the postictal state [26]. Altered monoamine receptor sensitivity has been supported by SPECT findings of low levels of striatal D2 in patients with peri-ictal psychosis [27]. GABAergic tone is central to seizure-related inhibitory mechanisms and its role in epilepsy-related psychoses is supported by demonstrable alterations in benzodiazepine receptors and favorable therapeutic response to benzodiazepines [28]. Similarly, there is strong evidence for the centrality of glutamatergic activity within an inhibition-excitation model of psychosis; NMDA receptor agonism is proconvulsant, NMDA receptor antagonism (with ketamine or PCP) is propsychotic and there is substantive evidence for glutamate receptor dysfunction in schizophrenia. Dopaminergic activity can also be considered within an excitation-inhibition paradigm with dopamine antagonism being proconvulsant and antipsychotic. Support for a kindling relationship between seizures and psychosis now includes a direct link in animal models between increased hippocampal activity in TLE 
and hyper-responsivity of the dopamine system. A significant increase in ventral tegmental dopamine neuron firing was observed after recurrent seizures in rats [29].

\section{Do specific antiepileptic drugs increase the risk of Psychoses in PWE?}

The long-standing debate with two sharply opposing hypotheses regarding the relationship between Psychoses in PWE and AEDs remains unresolved. Some authors have strongly advocated that any potent AED can precipitate psychosis precisely because of the potent anticonvulsant effects and that a subgroup of patients is particularly vulnerable to dramatic seizure reduction, irrespective of the AED used. In contrast, there are reports of psychotic symptoms specifically after exposure to certain AEDs [30,31,32,33] (including; ethosuximide [34], topiramate [35], felbamate [36], levetiracetam [37], zonisamide [38], vigabatrin [39], tiagabine [40] and lacosamide [41]. Whether they are specifically causal in the development of psychosis or not remains open to debate for reasons stated above. Psychosis related to lamotrigine remains controversial, with many studies unable to demonstrate an association [42].

Several authors have reported case-control studies or multiple case reports with special attention to AED-related psychosis. A summary of these studies is as follows. Recent modification of AEDs was found in 34 to $38 \%$ of PWE presenting with psychosis [30,31]; AED-related psychosis was more closely related to a decrease in seizure frequency in comparison with drug-independent psychosis [31,32] AED-related psychosis was more likely to be transient in comparison with drug-independent psychosis [30,31]. Medial temporal lobe involvement and genetic predisposition are overrepresented irrespective of the drug involved [32] in persons with AED-related psychosis. Frank psychosis has been reported more frequently following the initiation of specific AEDs including phenytoin, zonisamide, topiramate and levetiracetam when 
compared with other AEDs [31,32,37,42]. While these data appear conflicting prima facie, the disparity is resolved when the rarity of Psychosis in PWE responsive to first-line AED is accounted for. Even potent AEDs, which have a statistically greater tendency to be associated with psychosis when resulting in seizure freedom, do not typically cause psychosis in first-line AED treatment-responsive patients. Patients with epilepsy who fail to respond to second- or third-line AED treatment, or with a genetic predisposition to psychosis, are potentially more vulnerable to AEDs with negative psychotropic properties, which might precipitate an episode of psychosis. Finally, in the longer term, intractable epilepsy patients become psychotic with minimal change in epileptic activity, including not only AEDs but also surgical intervention or vagus nerve stimulation [43].

Psychosis could be a direct effect of the AED (pharmacological consequence, drug toxicity, drug withdrawal), or associated with the forced normalization phenomenon and its clinical counterpart, the alternating psychosis. Although speculative, psychosis as a complication of the administration of an AED may appear only in association with structural abnormalities and neurochemical dysfunctions involving the limbic circuit $[42,44]$.

Among the known associations of psychiatric symptoms in PWE, the use of AEDs is just one of the factors, alongside individual factors directly related to the patient and the specific individual epileptic disorder [33]. AEDs do not appear to be the single determinant of psychosis in PWE. Genetically determined individual susceptibility in conjunction with severity of the patient's epilepsy is probable additional factors [45]. Despite the lack of convincing evidence, in clinical practice it is advised that the aforementioned specific AEDs be used with caution in patients with a personal and/or 
positive family history of psychosis, patients with difficult to control epilepsy (e.g., TLE), particularly in high doses and or with rapid titration regimens [33].

\section{The problem of alternating psychosis}

Alternating psychoses, and the so-called "forced normalization" phenomenon, have been a matter of debate ever since the first reports of Heinrich Landolt during the 1950s.

Landolt reported EEG investigations of patients with epilepsy and paroxysmal psychiatric disorders, describing a group who had productive (i.e. with hallucinations) psychotic episodes with "forced normalization" of the EEG [46]. The abnormal EEGs of these patients improved or normalized during the time that they were psychotic. Subsequently, Tellenbach introduced the term "alternating psychoses" for the clinical phenomenon of the reciprocal relationship between abnormal mental states and seizures, which did not, as Landolt's term did, rely on EEG findings [47].

Since the early observations of Landolt, a number of patients with alternating psychosis have been documented, placing their existence beyond doubt. An association with the prescription of all AEDs was noted [48], suggesting that this is not a drug-specific phenomenon but rather linked to the neurobiological mechanisms underlying seizure control. A case of an alternating psychosis secondary to vagus nerve stimulation has been documented [49] and it is likely that the forced normalization phenomenon plays a role in patients who develop de novo psychosis following successful epilepsy surgery.

For many years, there has been vigorous debate on whether there is an epilepsy syndrome most commonly associated with the forced normalization phenomenon. Initially, drug-refractory focal epilepsies was claimed to be the prototype but subsequent literature on alternative psychoses seemed to favor generalized epilepsies [50]. In fact, in 
1985 Trimble found that idiopathic generalized epilepsy was most frequently associated with the forced normalization phenomenon [51]. Nowadays, there is general agreement that the forced normalization phenomenon occurs with both generalized and focal epilepsy, but is rare in patients with mainly generalized tonic-clonic seizures (e.g. idiopathic generalized epilepsy with tonic-clonic seizures of awakening) and in focal epilepsies outside the temporal lobe [48].

\section{Pharmacotherapy for brief and chronic interictal psychosis (IIP)}

Evidence for the treatment of IIP is limited, as evidenced by a recent Cochrane systematic review [52], which found only one small randomized controlled trial with insufficient power. Expert opinion is that antipsychotics and benzodiazepines are the treatment of choice for brief IIP [2]. Evidence on the relative efficacy of antipsychotics in brief IIP is also limited. In general, psychotic symptoms are better managed earlier rather than later [53]. When patients have mild psychotic symptoms or do not consent to psychopharmacological treatment, they can be offered psychosocial interventions, or be carefully monitored. One of the basic approaches to IIP episodes is to reduce AED polypharmacy and optimize the regimen [2]. When psychotic symptoms result in serious distress and/or psychosocial disturbance, psychopharmacological treatment is indicated.

The first step in the treatment of chronic IIP is accurate symptom detection and diagnosis [54]. In the early phase of an episode of IIP, it is difficult to predict the duration of the psychotic episode. Approximately $75 \%$ of all episodes of IIP last for one month or longer, which some authors have regarded as chronic [54]. Because the majority of episodes of IIP observed are recurrent, the boundary between the duration of 
brief and chronic subtypes is often drawn arbitrarily. Brief, in addition to chronic,

episodes of IIP may require longer-term APD treatment similar to that for primary

schizophrenia and should be considered long-term following remission [55].

\section{Pharmacotherapy for post-ictal psychosis (PIP)}

Florid episodes of PIP may require consideration of admission to a mental health unit and delay in therapeutic intervention may be associated with high risk behaviors to self and others $[56,57,58]$. Although the evidence is less compelling than in IIP, in both the initial and middle stages of PIP, sedative drugs are typically indicated, as they can abort or alleviate symptoms of PIP. Some experts have recommended benzodiazepines [59], while others prefer a combination of both benzodiazepines and antipsychotics [57]. In most cases, the authors' advise that effective sedation be prioritized as supported by Devinsky, 2008 [60].

In patients without severe symptoms, the administration of benzodiazepines may suffice. However, if increasing excitability emerges in the ensuing twenty-four hours, supplementary administration of antipsychotics may be necessary. In this clinical setting, benzodiazepines alone may precipitate paradoxical excitement and are not as potent as antipsychotics for rapid tranquillization of violent or agitated patients in a psychiatric emergency [61,62]. Paradoxically, ECT or repetitive transcranial magnetic stimulation have been reported as efficacious in terminating violent episodes of PIP in exceptional cases $[63,64]$.

\section{Efficacy, adverse events, and interactions of antipsychotic drugs (APDs) in PWE}

There is no evidence to support preferential initiation of any one specific APD in 
psychosis in PWE when equivalent doses are used [65], given the absence of randomized control trials specifically examining efficacy and tolerability of APDs in PWE. APDs should be selected with consideration of the balance between pharmacological profiles, efficacy, and adverse effects [2]. The clinical phenotype of psychotic phenomenon, which may vary considerably, might also warrant consideration [66]. APDs are predominantly effective in the symptomatic treatment of positive psychiatric symptoms, (e.g., delusions, hallucinations, formal thought disorder and bizarre behavior). They are less effective in the symptomatic treatment of negative psychiatric symptoms, (e.g., negative formal thought disorder, blunted affect, and apathy). In practice, atypical neuroleptics with few motor side-effects such as risperidone can be used as first choice: start low and go slow.

There are special considerations such as interactions with anticonvulsants, the profile of adverse events and multiple clinical phenotypes of the psychotic phenomenon $[66,67]$. In addition to possible proconvulsant effects of APD, as discussed later, clinicians must also consider the pharmacokinetic and pharmacodynamic interactions between anticonvulsants and antipsychotics [68]. Certain enzyme-inducing AEDs can increase the clearance of psychotropic drugs metabolized in the liver, including first-generation antipsychotics (chlorpromazine, fluphenazine and haloperidol) and atypical antipsychotics (including clozapine, olanzapine, risperidone (with the exception of phenytoin which inhibits risperidone clearance), quetiapine, aripiprazole and 
ziprasidone) [19]. In contrast, valproic acid has been reported to decrease the clearance of antipsychotic drugs (clozapine, olanzapine, quetiapine) [19]. Conversely, several psychotropic drugs can inhibit the clearance of some enzyme-inducing AEDs, in particular phenytoin and carbamazepine. These psychotropic drugs include some of the first-generation (haloperidol and loxapine) and second-generation (risperidone, quetiapine) APDs [19]. Carbamazepine reduces the plasma levels of all antipsychotics. This interaction is particularly evident for quetiapine which is predominantly metabolized by CYP 3A4 [69]. All of these interactions should be considered on an individual basis $[19,69]$.

The main adverse effects of APDs include sedation, weight gain and metabolic syndrome, cardiovascular effects, hyperprolactinemia, sexual dysfunction, and extrapyramidal symptoms. APD titration procedures are to start at a low dose, titrate gradually to a minimum therapeutic dose, and continue at a fixed therapeutic dose for a sufficient period of time. Patients with particular conditions, such as distinct brain damage and impaired hepatic/renal function, may require smaller initial doses with careful titration.

\section{Antipsychotics and seizure threshold}

Alper reported the rate of seizures associated with the use of APDs to range between $0.3 \%-3.5 \%$ among patients without epilepsy [70]. The entire class of APDs have been found associated with significantly increased seizure incidence ( $\mathrm{SIR}=2.05 ; 95 \% \mathrm{CI}$, 1.74-2.4). This finding remained significant after the removal of clozapine ( $\mathrm{SIR}=1.35$; 95\% CI, 1.09-1.66) but not after the removal of both clozapine and olanzapine (SIR= 
$1.18 ; 95 \%$ CI, .92-1.49), indicating that the observed increase was accounted for largely by clozapine and olanzapine [70]. Quetiapine was also associated with increased seizure incidence $(\mathrm{SIR}=2.05 ; 95 \% \mathrm{CI}, 1.21-3.23)$ relative to placebo. The remaining APDs, i.e. ziprasidone, aripiprazole and risperidone demonstrated no significant associated with seizure incidence $(\mathrm{SIR}=1.03 ; 95 \% \mathrm{CI}, .77-1.35)$ [70]. In clinical practice, is it possible to prescribe APDs in PWE without an increased risk in seizure frequency? Salient data include controlled studies in PWE, where the frequency of epileptic seizures was compared between two parallel groups, one on APDs and the other without; APD prescription was found not to increase seizure frequency [71]. Furthermore, the appearance of unusual EEG epileptiform activity and epileptic seizures with the introduction of clozapine has been evaluated [72]. Kikuchi et al. enrolled 26 patients with treatment resistant schizophrenia and reported that six patients prescribed clozapine experienced seizures (mean dose of clozapine at the first occurrence of seizure was 383 $\mathrm{mg} / \mathrm{d})$. Seizures were successfully treated with valproate or lamotrigine without discontinuation of clozapine. Successful treatment with clozapine without an increase in seizure frequency has been reported in a small case series [73] and case reports [74]. In clinical practice, the prescription of APDs does not increase the risk of seizures in PWE who are concurrently treated therapeutically with AEDs [75]. Transcranial magnetic 
stimulation (TMS) has been used to study cortical excitability changes in epilepsy

including the effect of AED use. A successful response to AED resulted in a decrease in cortical excitability and this effect was independent of the type of AED used [76]. The authors' clinical experience indicates that carefully administered APD treatment is safe for use in epilepsy. The aim should be to treat with the minimum effective dose. In clinically significant Psychoses in PWE, the benefits of APD prescription outweigh the increased theoretical probability of seizures in most cases [67].

\section{Conclusion}

The association between psychotic disorders and epilepsy remains incompletely understood. However, clinical experience and the limited systematic evidence available can be drawn on to set up a strategic framework for patient management. The proposed strategy is worthy of examination in controlled trials in the future. 


\section{References}

1. Trimble MR. The Psychoses of Epilepsy. New York: Raven Press, 1991

2. Adachi N, Kanemoto K, de Toffol B et al. Basic treatment principles for psychotic disorders in patients with epilepsy. Epilepsia 2013;54 (Suppl 1):19-33

3. Adachi N, Akanuma N, Fenwick P, et al. Seizure activity and individual vulnerability on first-episode interictal psychosis in epilepsy. Epil Behav $2018 ; 79: 234-8$

4. Logsdail SJ, Toone BK. Post-ictal psychoses: a clinical and phenomenological description. Br J Psychiatry 1988;152:246-52

5. Adachi N, Ito M, Kanemoto K et al. Duration of postictal psychotic episodes. Epilepsia $2007 ; 48: 1531-1537$

6. de Toffol B, Kanemoto K. Postictal psychoses: clinical and neurobiological findings. Encéphale $2016 ; 42: 443-447$

7. Trimble MR. The Intentional Brain: Motion, Emotion and the Development of Modern Neuropsychiatry. Baltimore: Johns Hopkins Univ. Press, 2016

8. Edeh J, Toone B. Relationship between interictal psychopathology and the type of epilepsy. Results of a survey in general practice. Br J Psychiatry 1987;151: $95-101$

9. Jalava M, Sillanpaa M. Concurrent illnesses in adults with childhood-onset epilepsy: a population-based 35-year follow-up study. Epilepsia $1996 ; 37: 1155-1163$

10. Stefansson SB, Olafsson E, Hauser WA. Psychiatric morbidity in epilepsy: a case 
controlled study of adults receiving disability benefits. $J$ Neurol Neurosurg Psychiatry 1998; 64:238-241

11. Gaitatzis A, Carroll K, Majeed A, Sander JW. The epidemiology of the comorbidity of epilepsy in the general population. Epilepsia 2004; 45:1613-1622

12. Forsgren L. Prevalence of epilepsy in adults in northern Sweden. Epilepsia 1992; $33: 450-458$

13. Clancy M.J., Clarke M.C., Connor D.J., et al. The prevalence of psychosis in epilepsy; a systematic review and meta-analysis. BMC Psychiatry 2014; 14: 75

14. Adelow C, Andersson T, Ahlbom A, et al. Hospitalization for psychiatric disorders before and after onset of unprovoked seizures/epilepsy. Neurology $2012 ; 78: 396-401$

15. Hesdorffer DC, Ishihara L, Maynepalli L et al. Epilepsy, suicidality, and psychiatric disorders: A bidirectional association. Ann Neurol 2012; 72: 184-191

16. Bredkjaer SR, Mortensen PB, Parnas J. Epilepsy and non-organic non-affective psychosis. National epidemiologic study. Br J Psychiatry 1998; 172:235-238

17. Qin P, Xu H, Laursen TM, et al. Risk for schizophrenia and schizophrenia-like psychosis among patients with epilepsy: population based cohort study. $B M J$ 2005;331: 23

18. Thorup A, Waltoft BL, Pedersen CB, et al. Young males have a higher risk of developing schizophrenia: a Danish register study. Psychol Med 2007; $37: 479-484$

19. Kanner AM. Management of psychiatric and neurological comorbidities in epilepsy. Nat Rev Neurol 2016;12:106-16 
20. Irwin LG, Fortune DG. Risk factors for psychosis secondary to Temporal Lobe Epilepsy: A systematic Review. J Neuropsychiatry Clin Neurosci 2014; 26:5-23

21. Schulze-Bonhage A, van Elst LT. Postictal psychosis: Evidence for extrafocal functional precursors. Epilepsy Behav 2010; 18 :308-312

22. Suzuki M. Differential contributions of prefrontal and temporolimbic pathology to mechanisms of psychosis. Brain 2005; 128:2109-2122

23. Fong GCY. Postictal psychosis related regional cerebral hyperperfusion. J Neurol Neurosurg Psychiatry 2000; 68:100-101

24. Oshima T, Motooka H, Kanemoto K. SPECT findings during postictal psychoses: predominance of relative increase of perfusion in right temporal lobe. Epilepsia 2011;52:1192-4

25. Butler T, Weisholtz D, Isenberg N et al. Neuroimaging of frontal-limbic dysfunction in schizophrenia and epilepsy-related psychosis: Toward a convergent neurobiology. Epilepsy Behav 2012; 23:113-122

26. Sachdev P. Schizophrenia-Like psychosis and epilepsy: the status of the association. Am J Psychiatry1998; 155:325-336

27. Ring HA, Trimble MR, Costa DC, Moriarty J, Verhoeff NPLG, Ell PJ. Striatal dopamine receptor binding in epileptic psychoses. Biol Psychiatry 1994; $35: 375-380$

28. Sachdev PS. Alternating and postictal psychoses: review and a unifying hypothesis. Schizophr Bull 2007; 33:1029-1037

29. Cifelli P, Grace A. Psychosis in temporal lobe epilepsy and the modulation of dopamine system activity. Biol Psychiatry 2009; 64(Suppl) :S26

30. Kanemoto K, Tsuji T, Kawasaki J. Re-examination of interictal psychoses; 
based on DSM IV psychosis classification and international epilepsy classification. Epilepsia 2001;42:98-103

31. Matsuura M. Epileptic psychoses and anticonvulsant drug treatment. $J$ Neurol Neurosurg Psychiatry 1999; 67:231-3

32. Mula M, Trimble MR, Sander JW. Are psychiatric adverse events of antiepileptic drugs a unique entity? A study on topiramate and levetiracetam. Epilepsia 2007;48:2322-6

33. Mula M., Monaco F. Antiepileptic drugs and psychopathology of epilepsy: an update. Epileptic Disord 2009; 11: 1-9

34. Goren M, Onat F. Ethosuximide: from bench to bedside. CNS Drug Rev 2007; 13 : $224-39$

35. Mula M, Trimble MR, Lhatoo SD, Sander JW. Topiramate and psychiatric adverse events in patients with epilepsy. Epilepsia 2003; 44: 659-63

36. Knable MB, Rickler K. Psychosis associated with felbamate treatment. J Clin Psychopharmacol 1995;15: 292-3

37. Chen Z, Lusicic A, O'Brien TJ, Velakoulis D, Adams SJ, Kwan P. Psychotic disorders induced by antiepileptic drugs in people with epilepsy. Brain 2016; 139: $2668-78$

38. Matsuura M, Trimble MR. Zonisamide and psychosis. J Epilepsy 1997; 10: 52-4

39. Weber P, Dill P, Datta AN. Vigabatrin-induced forced normalization and psychosis prolongated termination of behavioral symptoms but persistent antiepileptic effect after withdrawal. Epilepsy 
Behav 2012; 24: 138-40

40. Sackellares JC, Krauss G, Sommerville KW, Deaton R. Occurrence of psychosis in patients with epilepsy randomized to tiagabine or placebo treatment. Epilepsia 2002; 43: 394-8

41. Pinkhasov A, Lam T, Hayes D, Friedman M, Singh D, Cohen H. Lacosamide induced psychosis: case Report, review of differential diagnosis and relevant pharmacokinetics. Clin Neuropharmacol 2015; 38: 198-200

42. Noguchi T, Fukatsu N, Kato H, Oshima T, Kanemoto K. Impact of antiepileptic drugs on genesis of psychosis. Epilepsy Behav 2012;23:462-5

43. Kanner AM. Psychosis of epilepsy: a neurologist's perspective. Epilepsy Behav $20001: 219-227$

44. Matsuura M. Antiepileptic drugs and psychosis in epilepsy. In: Matsuura M, Inoue Y. Neuropsychiatric Issues in Epilepsy. Montrouge: John Libbey Eurotext 2010:13-25

45. Turan AB, Seferoglu M, Taskapilioglu O et al. Vulnerability of an epileptic case to psychosis: sodium valproate with lamotrigine, forced normalization, postictal psychosis or all ? Neurol Sci 2012; 33:1161-3

46. Landolt H. Serial EEG investigations during psychotic episodes in epileptic patients and during schizophrenic attacks. In: Lorenz de Haas AM. Lectures on Epilepsy. Amsterdam: Elsevier, 1958, 91-133

47. Tellenbach H. Epilepsie als anfallsleiden und als psychose. Uber alternative psychosen paranoider pragung bei "forcierte Normalisierung" (Landolt) des elektroenzephalogramms epileptischer. Nervenarzt 1965;36:190-202

48. Mula M. The Landolt's phenomenon: an update. Epileptologia 2010;18:39-44 
49. Gatzonis SD, Stamboulis E, Siafakas A et al. Acute psychosis and EEG normalisation after vagus nerve stimulation. J Neurol Neurosurg Psychiatry 2000; $69: 278-9$

50. Trimble MR, Schmitz B. Forced normalization and alternative psychoses of epilepsy. Petersfield: Wrightson Biomedical Publishing Ltd, 1998

51. Wolf P, Trimble MR. Biological antagonism and epileptic psychosis. $B r J$ Psychiatry 1985;146:272-6

52. Farooq S, Sherin A. 2015. Interventions for psychotic symptoms concomitant with epilepsy. Cochrane Database Syst Rev, CD006118

53. Adachi N, Akanuma N, Ito M, Okazaki M, Kato M, Onuma T. Interictal psychotic episodes in epilepsy: duration and associated clinical factors. Epilepsia 2012;53: 1088-94

54. Adachi N, Akanuma N. Delusions and hallucinations. In: Mula M. Neuropsychiatric symptoms of epilepsy. New York: Springer International Publishing, 2015:69-89

55. Kerr M, Mensah MP, Besag, F et al. International consensus clinical practice statements for the treatment of neuropsychiatric conditions associated with epilepsy. Epilepsia 2011;52, 2133-8

56. Devinsky O, Abramson H, Alper K et al. Postictal psychosis: a case control series of 20 patients and 150 controls. Epilepsy Res 1995; 20: 247-53

57. Kanner AM, Stagno S, Kotagal P, Morris HH. Postictal psychiatric events during prolonged video-electroencephalographic monitoring studies. Arch Neurol $1996 ; 53: 258-263$ 
58. Kanemoto K, Kawasaki J, Kawai I. Postictal psychosis: a comparison with acute interictal and chronic psychoses. Epilepsia 1996; 37: 551-6

59. Lancman ME, Craven WJ, Asconapé JJ, Penry JK. Clinical management of recurrent postictal psychosis. J Epilepsy 1994;7:47-51

60. Devinsky O. Postictal psychosis : common, dangerous, and treatable. Epilepsy Curr $2008 ; 8: 31-4$

61. Alexander J, Tharyan P, Adams C, John T, Mol C, Philip J. Rapid tranquillisation of violent or agitated patients in a psychiatric emergency setting: pragmatic randomised trial of intramuscular lorazepam vs haloperidol plus promethazine. $\mathrm{Br}$ J Psychiatry 2004;185:63-69

62. Allen MH, Currier GW, Carpenter D et al. The expert consensus guideline series. Treatment of behavioral emergencies 2005. J Psychiatr Pract 2005; 11 (Suppl1):5

63. Pascual-Aranda A, Garcia-Morales I, Sanz-Fuentenebro J. Postictal psychosis: resolution after electroconvulsive therapy. Epilepsy Behav 2001; 2: 363-366

64. Krauss G, Theodore WH. Treatment strategies in the postictal state. Epilepsy Behav 2010; 19:188-90

65. Hara K, Adachi N, Okazaki M et al. Duration of interictal psychotic episodes by antipsychotic drug treatments. Epilepsy Behav 2013;27:342-345

66. Gaitatzis A, Trimble, MR, Sander JW 2004. The psychiatric comorbidity of epilepsy. Acta Neurol Scand 2004;110: 207-20

67. Koch-Stoecker S. Antipsychotic drugs and epilepsy: indications and treatment guidelines. Epilepsia 2002;43 (Suppl 2): 19-24

68. LaFrance W.C. JR., Kanner A. M., Hermann B. Psychiatric comorbidities in 
epilepsy. Int Rev Neurobiol 2008;83: 347-83

69. Mula M. The pharmacological management of psychiatric comorbidities in patients with epilepsy. Pharmacol Res 2016;107:143-57

70. Alper K, Schwartz KA, Kolts RL, Khan A. Seizure incidence in psychopharmacological clinical trials: An analysis of Food and Drug Administration (FDA) summary basis of approval reports. Biol Psychiatry $2007 ; 62: 345-354$

71. Okazaki M, Adachi N, Hara K, Ito M, Kato M, Onuma T. Do antipsychotic drugs increase seizure frequency in epilepsy patients? Eur Psychoneuropharmacol 2014;24:1738-44

72. Kikuchi YS, Sato W, Ataka K, et al. Clozapine-induced seizures, electroencephalography abnormalities and clinical responses in Japanese patients with schizophrenia. Neuropsychiatr Dis Treat 2014;10:1973-8

73. Langosch JM, Trimble MR. Epilepsy, psychosis and clozapine. Hum Psychopharmacol 2002;17:115-9

74. Jetté Pomerleau V, Dubeau F, Ducharme S. Clozapine safety and efficacy for interictal psychotic disorder and pharmacoresistant epilepsy. Cogn Behav Neurol $2017 ; 2: 73-6$

75. Pisani F, Oteri G, Costa C, Di Raimando G, Di Perri R. Effects of psychotropic drugs on seizure threshold. Drug Safety 2002;25:91-110

76. Badawy RAB, Macdonell RAL, Berkovic SF, Newton MR, Jackson GD. Predicting seizure control: cortical excitability and antiepileptic medication. Ann Neurol 2010;67:64-73 